\title{
The Dorsomedial Pathway Is Not Just for Reaching: Grasping Neurons in the Medial Parieto-Occipital Cortex of the Macaque Monkey
}

\author{
Patrizia Fattori, ${ }^{1}$ Vassilis Raos, ${ }^{2}$ Rossella Breveglieri, ${ }^{1}$ Annalisa Bosco, ${ }^{1}$ Nicoletta Marzocchi, ${ }^{1}$ and Claudio Galletti ${ }^{1}$ \\ ${ }^{1}$ Dipartimento di Fisiologia Umana e Generale, Università di Bologna, I-40126 Bologna, Italy, and ${ }^{2}$ Department of Basic Sciences, School of Health Sciences, \\ University of Crete, GR-71003 Iraklion, Greece
}

\begin{abstract}
Brain control of prehension is thought to rely on two specific brain circuits: a dorsomedial one (involving the areas of the superior parietal lobule and the dorsal premotor cortex) involved in the transport of the hand toward the object and a dorsolateral one (involving the inferior parietal lobule and the ventral premotor cortex) dealing with the preshaping of the hand according to the features of the object. The present study aimed at testing whether a pivotal component of the dorsomedial pathway (area V6A) is involved also in hand preshaping and grip formation to grasp objects of different shapes.

Two macaque monkeys were trained to reach and grasp different objects. For each object, animals used a different grip: whole-hand prehension, finger prehension, hook grip, primitive precision grip, and advanced precision grip. Almost half of 235 neurons recorded from V6A displayed selectivity for a grip or a group of grips. Several experimental controls were used to ensure that neural modulation was attributable to grip only.

These findings, in concert with previous studies demonstrating that V6A neurons are modulated by reach direction and wrist orientation, that lesion of V6A evokes reaching and grasping deficits, and that dorsal premotor cortex contains both reaching and grasping neurons, indicate that the dorsomedial parieto-frontal circuit may play a central role in all phases of reach-to-grasp action. Our data suggest new directions for the modeling of prehension movements and testable predictions for new brain imaging and neuropsychological experiments.
\end{abstract}

\section{Introduction}

Reaching out to grasp an object requires a plethora of complex brain operations to control the transport of the hand toward the object, the orientation of the wrist to match the object orientation, and the preshaping of the fingers into a grip appropriate for the shape and size of the object. The transport phase is mainly accomplished with the proximal musculature of the forelimb and the grasping phase with the distal musculature.

In the early 1980s, it was suggested that the action of reaching to pick up an object could be partitioned into distinct "transport" and "grasp" components, each controlled by an identifiable system characterized by its own inputs, outputs, and intrinsic wiring (Arbib, 1981; Jeannerod, 1981). Presumably, there would also have to be a mechanism for achieving coordination between the two components, given that they mesh together to enable a seamless unfolding of the skilled prehension movement (for review, see Jeannerod, 1997). The two-system theory has led to a proposed embodiment of the separate components in two specific

Received Aug. 4, 2009; revised Sept. 10, 2009; accepted 0ct. 23, 2009

This research was supported by European Union Grant FP6-IST-027574 (MATHESIS) and by Ministero dell'Università e della Ricerca and Fondazione del Monte di Bologna e Ravenna, Italy. We are grateful to Daniel Bacher for developing the permutation test, Lefteris Neromiliotis for helping in performing the quantitative analyses, and Stuart Baker for helpful advice in setting up the multielectrode recording system.

Correspondence should be addressed to Patrizia Fattori, Dipartimento di Fisiologia Umana e Generale, Università di Bologna Piazza di Porta San Donato 2, I-40126 Bologna, Italy. E-mail: patrizia.fattori@unibo.it.

DOI:10.1523/JNEUROSCI.3800-09.2010

Copyright $\odot 2010$ the authors $\quad 0270-6474 / 10 / 300342-08 \$ 15.00 / 0$ parieto-frontal pathways: a medial one for reaching, encompassing the superior parietal lobule (SPL) and the dorsal premotor cortex (Caminiti et al., 1996; Jeannerod, 1997; Wise et al., 1997), and a lateral one for grasping, involving the inferior parietal lobule and the ventral premotor cortex (Jeannerod et al., 1995; Jeannerod, 1997; Luppino et al., 1999). Although the theory of parallel channels has been debated and criticized by several authors from both experimental and theoretical points of view (Desmurget et al., 1996; Smeets and Brenner, 1999; Mon-Williams and McIntosh, 2000), this proposed division of labor has become firmly established and is accepted by influential and widely used textbooks of neuroscience (Krakauer and Ghez, 2000).

In the past decade, it has been demonstrated that a visuomotor area within the dorsomedial visual stream, located in the caudalmost part of the SPL (area V6A) (see Fig. 1a) (Galletti et al., 1999, 2003), contains "reaching" neurons able to encode the direction of the arm toward different spatial locations (Fattori et al., 2001 , 2005). Recently, it has been reported that V6A neurons are also modulated by the orientation of the wrist during reach-tograsp actions (Fattori et al., 2009). The aim of the present study was to investigate whether V6A neurons are also modulated by the most distal component of prehension, that is, the preshaping of the hand to form the correct grip for grasping an object. Our results demonstrate for the first time that the caudal SPL, a critical node within the dorsomedial pathway, contains neurons that code the different types of grip used for grasping different objects. These new data, together with those of our previous studies 
(Fattori et al., 2001, 2009), demonstrate that area V6A contains neurons whose responses are sensitive to all phases of a reach-tograsp action. The discovery of grasping neurons in the dorsomedial visual stream forces a rethinking of our ideas as to the cerebral circuitry involved in planning and controlling prehension movements in the primate brain.

\section{Materials and Methods}

Behavioral task. The monkey sat in a primate chair (Crist Instruments) with the head fixed, in front of a personal computer-controlled panel containing five different objects. The objects were presented to the animal one at a time, in a random order. During the intertrial period, the panel was reconfigured by the personal computer so as to present the object to be grasped on the next trial in the same position $(22.5 \mathrm{~cm}$ away from the animal, in the midsagittal plane). View of the remaining four objects was occluded.

The objects and the grip types used for grasping them are illustrated in Figure $1 c$. The grasping axis of the objects could be oriented either horizontally or vertically. The types of grip evoked by the objects varied according to their physical characteristics and were as follows: whole-hand prehension, with all the fingers wrapped around the "ball" (diameter of $30 \mathrm{~mm}$ ) and with the palm in contact with the object; finger prehension, by inserting all the fingers (but not the thumb) into the gap behind the "handle" (gap dimensions, $28 \times 11 \times 2 \mathrm{~mm}$ ); hook grip, in which the index finger was inserted into the "ring" (internal diameter, $12 \mathrm{~mm}$; external diameter, $17 \mathrm{~mm}$ ); primitive precision grip, using the thumb and the distal phalanges of the other fingers to grasp the "plate" (width, $30 \mathrm{~mm}$; length, $14 \mathrm{~mm}$; thickness, $4 \mathrm{~mm}$ ); and advanced precision grip, with the pulpar surface of the last phalanx of the index finger opposed to the pulpar surface of the last phalanx of the thumb, to grasp the "stick-in-groove" (cylinder with base diameter of $10 \mathrm{~mm}$ and length of $11 \mathrm{~mm}$, in a slot $12 \mathrm{~mm}$ wide, $15 \mathrm{~mm}$ deep, and $30 \mathrm{~mm}$ long).

The reach-to-grasp task, illustrated in Figure $1 b$, had the following time sequence: the trial began when the monkey pressed a "home" button near its chest, outside its field of view, in complete darkness. The animal was required to keep the button pressed for $1 \mathrm{~s}$, during which it was free to look around, though remaining in darkness. After this interval, a light-emitting diode (LED) mounted above the object was switched on and the monkey had to fixate it. Breaking of fixation and/or premature button release interrupted the trial. After a delay period of $0.5-1 \mathrm{~s}$, two lateral lights were turned on and the object was illuminated for a period of $0.5 \mathrm{~s}$. The lights were then switched off for the rest of the trial. After a second delay period of $0.5-2 \mathrm{~s}$ during which the monkey was required to keep fixation on the LED without releasing the home button, the color of the LED changed. This was the go-signal for the monkey to release the button and perform a reach-to-grasp movement toward the object, pull it, and hold it until the LED was switched off (after $0.8-1.2 \mathrm{~s}$ ). The LED switch-off cued the monkey to release the object and to press the home button again. Button pressing ended the trial, allowed the monkey reward, and started another trial, in which another object, randomly chosen, was presented.

As described above, the monkey could see the object only briefly at the beginning of the trial, when no arm movement was allowed, and the subsequent reach-to-grasp action was executed in complete darkness. In this way, the monkey could accomplish the reach-to-grasp movement adapting the grip to the object shape on the basis of the visual information it received at the beginning of each trial, well before the go signal.

When the acquisition of a neuron was achieved, the background light was switched on for a few minutes to avoid dark adaptation. The dark environment excluded any modulation of cell activity attributable to visual stimulation evoked by arm movements in the visual field. In addition, the brightness of the fixation LED (masked by a $1.5 \mathrm{~mm}$ aperture) was lowered so as to be barely visible during the task (standing by the monkey, the experimenter could not see the object or the monkey's hand moving in its peripersonal space, even while dark adapted).

Monkeys performed the reach-to-grasp movements with the arm contralateral to the recording side. Monkeys' hand movements were continuously video monitored by means of miniature video cameras sensitive to infrared illumination. The trajectories of monkey arm movements performed during the task were estimated using video images at 25 frames $/ \mathrm{s}$. To inspect proximal joint trajectories during reaching for the different five ob-
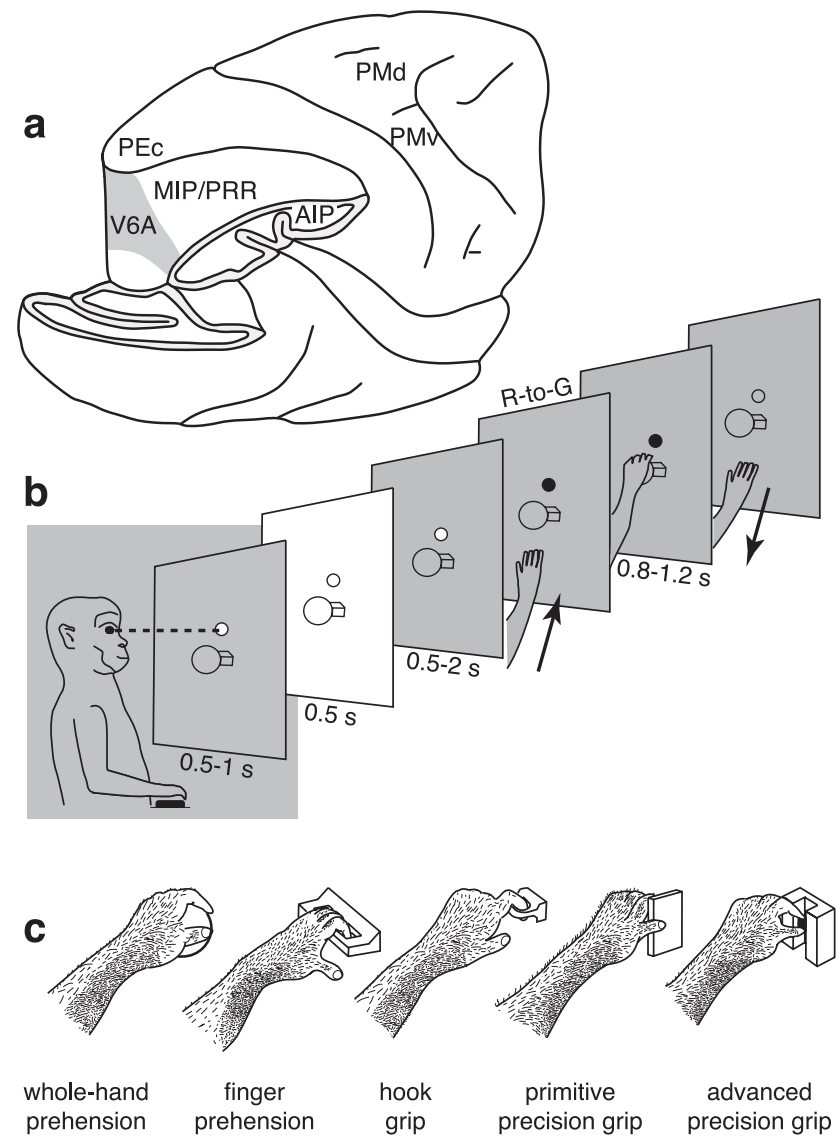

Figure 1. Recording site, reach-to-grasp task, and grip types. $\boldsymbol{a}$, Brain silhouette with part of the occipital pole and inferior parietal lobule cut away to show the location of the recording site (area V6A, gray shaded) in the anterior bank of the parieto-occipital sulcus. Locations of other parietal and frontal areas are also indicated. $\boldsymbol{b}$, Time course of the reach-to-grasp task. The animal was trained to fixate at a constant location (fixation LED) shown as a small circle in front of the animal. It reached for and grasped an object (a ball, in this example) visible only at the beginning of the trial (white panel). The R-to-G action was executed in darkness, as indicated by the gray shading. $c$, Drawing (derived from video frames) of the five objects and grip types used by the monkey. The object to be grasped changed from trial to trial, so requiring different hand preshaping for the accomplishment of the grip. The orientation of the objects was chosen so that wrist orientation was similar in all cases. The five objects were grasped with five different grips: the ball with the whole hand (leftmost drawing), the handle with fingers only, the ring with the index finger only (hook grip), the plate with a primitive precision grip with fingers/thumb opposition, and the stick-in-groove with an advanced precision grip with precise index-finger/thumb opposition. PEC, Area PE caudal; PMd, dorsal premotor cortex; PMv, ventral premotor cortex; PRR, parietal reach region.

jects, the relevant points related to shoulder and elbow joints (corresponding to the lesser tuberosity of the humerus and the lateral epicondyle, respectively) were identified on single video frames and traced along the trial.

The behavioral task was controlled by custom-made software implemented in a Labview Realtime environment (Kutz et al., 2005). Trials in which the monkey's behavior was incorrect were discarded. Eye position was monitored through an infrared oculometer system (Voss Eyetracker).

Experimental procedures. Experiments were performed in accordance with national laws on care and use of laboratory animals and with the European Communities Council Directive of November 24, 1986 (86/609/EEC). During training and recording sessions, particular attention was paid to any behavioral and clinical sign of pain or distress. Experiments were approved by the Bioethical Committee of the University of Bologna.

Two Macaca fascicularis monkeys, weighing 3650 and 2450 g, were trained to perform the reach-to-grasp task described above. The monkeys were trained for 5-6 months to reach a performance level $>95 \%$. The head-restraint system and the recording chamber were surgically implanted in asepsis and under general anesthesia (sodium thiopenthal, $8 \mathrm{mg} \cdot \mathrm{kg}^{-1} \cdot \mathrm{h}^{-1}$, i.v.) following the procedures reported by Galletti et 
al. (1995). Adequate measures were taken to minimize pain or discomfort. A full program of postoperative analgesia (ketorolac trometazyn, 1 $\mathrm{mg} / \mathrm{kg}$, i.m. immediately after surgery and $1.6 \mathrm{mg} / \mathrm{kg}$, i.m. on the following days) and antibiotic care [Ritardomicina (benzatinic benzylpenicillin plus dihydrostreptomycin plus streptomycin) $1-1.5 \mathrm{ml} / 10 \mathrm{~kg}$ every $5-6$ d] followed the surgery.

Single-cell activity was recorded extracellularly from area V6A. We performed single microelectrode penetrations using home-made glasscoated metal microelectrodes with a tip impedance of $0.8-2 \mathrm{M} \Omega$ at $1 \mathrm{kHz}$ and multiple electrode penetrations using a five-channel multielectrode recording minimatrix (Thomas Recording). The electrode signals were amplified (at a gain of 10,000) and filtered (bandpass between 0.5 and 5 $\mathrm{kHz}$ ). Action potentials in each channel were isolated with a dual timeamplitude window discriminator (DDIS-1; Bak Electronics) or with a waveform discriminator (Multi Spike Detector; Alpha Omega Engineering). Spikes were sampled at $100 \mathrm{kHz}$, and eye position was simultaneously recorded at $500 \mathrm{~Hz}$. Location of area V6A was initially identified on functional grounds during recordings (Galletti et al., 1999) and later confirmed following the cytoarchitectonic criteria of Luppino et al. (2005).

Statistical analysis. The influence of grasping on V6A neuronal activity was analyzed in the reach-to-grasp (R-to-G) epoch, which lasts from 200 $\mathrm{ms}$ before the movement onset (button release) up to the movement end (object pulling). A neuron was considered as grasp related when it displayed a statistically significant difference in activity between the epoch FREE (from button press to LED switch-on) and epoch R-to-G and also between epochs R-to-G when using different types of grip [grip (5 levels) $\times$ epoch (2 levels), two-way ANOVA, $p<0.05$ ]. Statistical analyses were performed using the Statistica software (StatSoft). We analyzed only those units tested for five grips in at least seven trials for each grip. The reasons for these conservative criteria are dictated by the intrinsic high variability of biological responses.

Population response was calculated as averaged spike density function (SDF). An SDF was calculated (Gaussian kernel, half-width of $40 \mathrm{~ms}$ ) for each neuron included in the analysis and averaged across all the trials for each tested grip. The peak discharge of the neuron found over all grip types during the R-to-G epoch was used to normalize all the SDFs for that neuron. The normalized SDFs were then averaged to obtain population responses (Marzocchi et al., 2008). To statistically compare the SDFs of the various grip types (see Figs. 3, 5), we performed a permutation test using the sum of squared error as the test statistic. For each pair of grip types, the two sets of curves were randomly permuted, and the resulting sum of squared error was compared with the actual one over 10,000 repetitions. Because the weighted mean of the movement time was $574.7 \pm 100.9 \mathrm{~ms}$, comparisons have been made in the interval from 200 $\mathrm{ms}$ before movement onset to $800 \mathrm{~ms}$ after it.

To quantify the preference of the recorded neurons for the different types of grip, we computed a preference index (PI) that takes into account the magnitude of the neuronal response to each of the five grips. According to Moody and Zipser (1998), it was computed as follows:

$$
\mathrm{PI}=\frac{n-\left(\frac{\sum r_{i}}{\mathrm{r}_{\text {pref }}}\right)}{n-1},
$$

where $n$ is the number of objects, $r_{i}$ is the activity for object $i$, and $r_{\text {pref }}$ is the activity for the preferred object in the R-to-G epoch. The PI can range between 0 and 1.0. A value of 0 indicates the same magnitude of response for all five grips, whereas a value of 1 indicates a response for only one grip type.

The percentage discharge difference between best and second best grip was computed as $\left(r_{\text {best }}-r_{\text {second best }}\right){ }^{*} 100 / r_{\text {best }}$. Similarly, the best-worst difference was estimated as $\left(r_{\text {best }}-r_{\text {worst }}\right){ }^{\star} 100 / r_{\text {best }}$. All the analyses were performed using custom scripts in Matlab (MathWorks).

To assess how the neurons encode the similarity among the five grip types, we performed hierarchical cluster analysis using SPSS software. For this analysis, the average firing rate for each grip was taken into account (see Fig. 6). At the first step of the hierarchical cluster analysis, each grip represents its own cluster and the similarities between the grips are defined by a measure of cluster distance. In the present study, we used the squared Euclidean distance, which places progressively greater weight on grips that are farther apart. On the basis of the calculated distance, the two closest clusters are merged to form a new cluster replacing the two old clusters. The distances between these new clusters are determined by a linkage rule. In the present study, the complete linkage rule has been used. For this method, the dissimilarity between cluster A and cluster B is represented by the maximum of all possible distances between the cases in cluster $A$ and the cases in cluster B. The steps in a hierarchical clustering solution that shows the clusters being combined and the values of the distance coefficients at each step are shown by dendrograms. Connected vertical lines designate joined cases. The dendrogram rescales the actual distances to numbers between 0 and 25, preserving the ratio of the distances between steps.

\section{Results}

We recorded from 235 neurons in area V6A of two macaque monkeys trained to perform a reach-to-grasp task under controlled conditions. The task required the monkey to transport the hand from a position near the body to a fixed position in peripersonal space where an object was presented. The object had to be grasped in darkness after a brief period of visual presentation. The time sequence of the task is depicted in Figure $1 b$. A different object was presented on each trial, each object requiring a different grip type. Figure $1 c$ shows the objects tested and the grips necessary to grasp them. Grasping the ball required a whole-hand prehension; the handle required the fingers to be inserted into the gap and wrapped around the handle after aligning the wrist with the handle orientation; the ring required a "hook grip" using the index finger; the plate required a primitive precision grip with fingers/thumb opposition; and the stick-in-groove, an advanced precision grip using index finger/thumb opposition.

One hundred eleven cells ( $47 \%$ of the total) displayed grip selectivity in the reach-to-grasp epoch (Fig. 1b, R-to-G), as assessed by two-way ANOVA (factor 1, grip; factor 2, epoch; $p<$ $0.05)$. V6A neural discharges during the execution of the reachto-grasp actions are the focus of this work. Figure 2 shows examples of four grasp-related neurons.

The response of neuron A152 (Fig. 2b) begins before the onset of the reach-to-grasp movement; it rises rapidly during the approach of the hand toward the object, peaks at approximately midway through the transport phase, and returns to baseline as soon as the object is grasped and held in the monkey's hand. The temporal pattern of discharge is similar for the five grips tested, but the intensity of the response is modulated by the grip type performed by the monkey. This neuron displays a rather broad selectivity for grips. It discharges intensively during the execution of the advanced precision grip and hook grip, and the firing rate is lower during the primitive precision grip and significantly less during the execution of the whole-hand and finger prehension $\left(F_{(4,45)}=6.697, p<0.001\right)$. The activity of this cell during the formation of the handgrip seems to reflect the use of the index finger. The control of this finger is crucial for the correct performance of the preferred grips of a neuron.

Also, the cell presented in Figure $2 c$ is broadly tuned to grasp execution: the firing rate is maximum when the animal performs finger prehension; it progressively reduces for the other types of grip, arriving at a minimum for the primitive precision grip $\left(F_{(4,45)}=12.130, p<0.001\right)$. The neurons in Figure $2, d$ and $e$, are much more selective for grip type than the neurons in Figure 2, $b$ and $c$. Neuron B243 (Fig. $2 d$ ) is maximally activated during the execution of the advanced precision grip $\left(F_{(4,45)}=7.038, p<\right.$ 0.001 ), i.e., when the animal used the most skilled grip (opposition of the distal phalanges of the index and of the thumb). Neuron A101 (Fig. 2e) discharges maximally during the execution of the most rudimentary grip, i.e., the whole-hand prehension $\left(F_{(4,36)}=54.038, p<0.001\right)$. 


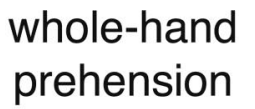

a
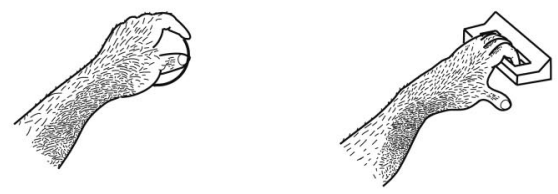

b
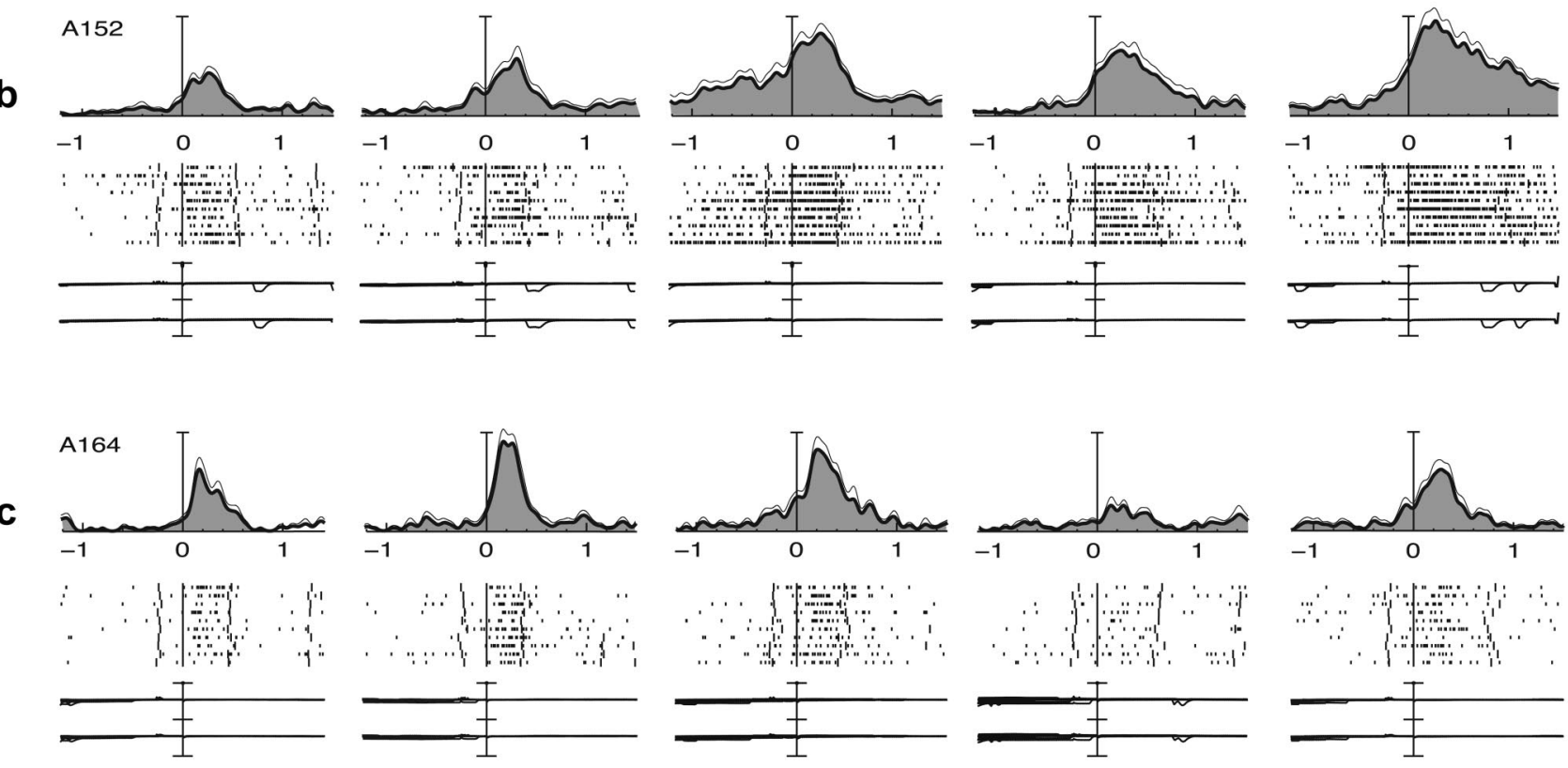

B243

d
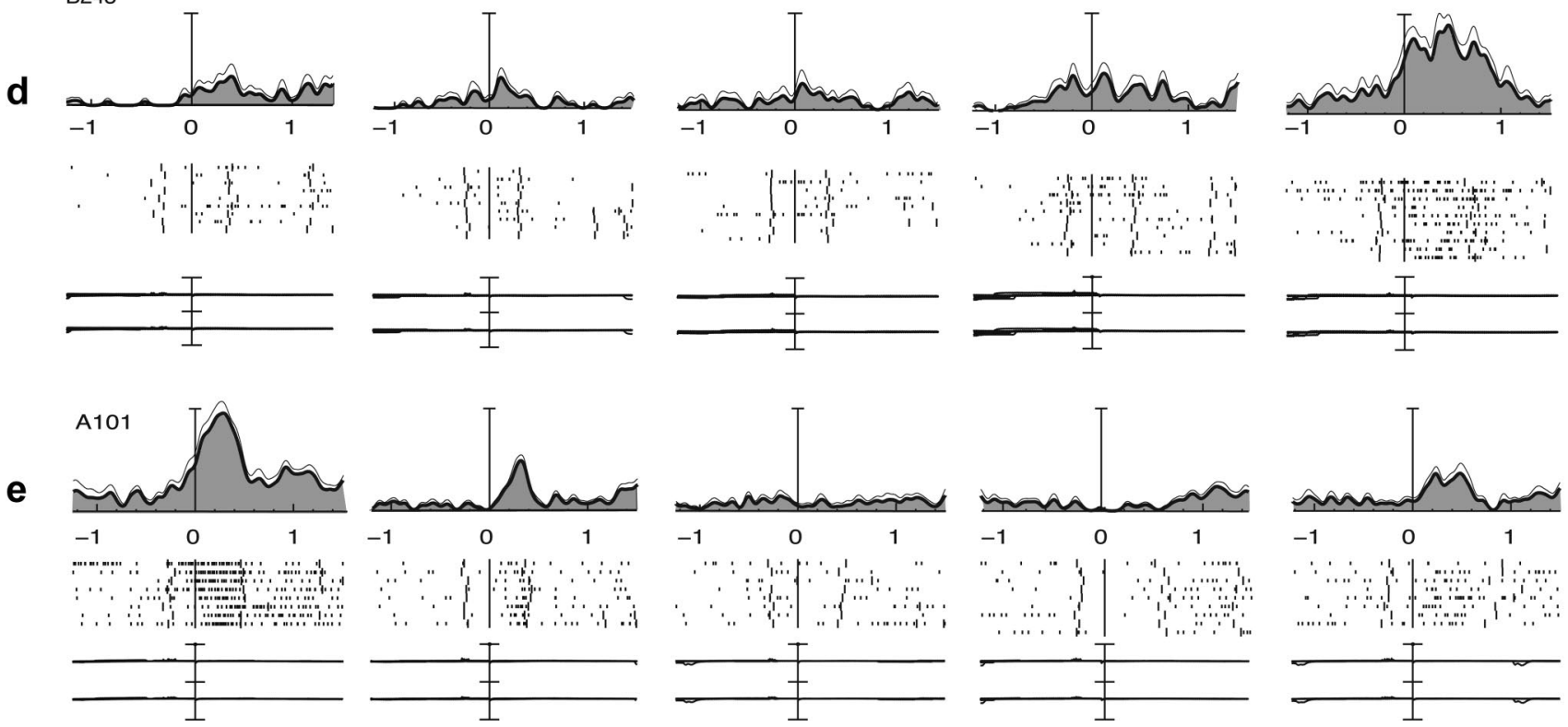

Figure 2. Examples of V6A grasping neurons. $\boldsymbol{a}$, Types of grip used by the monkeys to grasp the different objects. $\boldsymbol{b}$ - $\boldsymbol{e}$, Examples of V6A grasping neurons. Neuronal activity is expressed as SDFs (thick lines) with variability band (SEM, light lines) and raster displays of impulse activity. Neural activity and recordings of $x$ and $y$ components of eye position are aligned on the onset of the reach-to-grasp movement. Long vertical ticks in raster displays are behavioral markers, indicating the following, from left to right: go signal (LED color change), onset of movement (button release), object pull, cue to release the object, and object release. All four cells shown in the figure discharge during hand preshaping en route toward the object (reach-to-grasp epoch) and display different grip preferences and different levels of selectivity. $\boldsymbol{b}$ and care broadly selective, and $\boldsymbol{d}$ and $\boldsymbol{e}$ are highly selective. Vertical scale bars on histograms: $\boldsymbol{b}, 125 \mathrm{spikes} / \mathrm{s} ; \boldsymbol{c}, 65 \mathrm{spikes} / \mathrm{s} ; \boldsymbol{d}, 35 \mathrm{spikes} / \mathrm{s} ; \boldsymbol{e}, 70$ spikes/s. Eye traces: $60 \%$ division. 
From the neurons shown in Figure 2, it is evident that different times are required for the accomplishment of the different grips. This may lead one to suggest that movement duration could be a factor affecting neurons discharge, and this could contribute to explain the discharge differences observed among the various grips. To investigate this issue, in each grasping neuron, we performed regression analysis between the movement times for the various grips and the corresponding discharge frequencies. All neurons but two displayed not statistically significant correlations $(p>$ 0.01 ) demonstrating that movement duration does not contribute to the grip specificity displayed by the neurons.

It is worth noting that the monkey executed the reach-tograsp movement in complete darkness without being able to see the hand, nor the object, nor their interaction (Fig. $1 b$ ), so the modulation of cell discharge cannot be ascribed to visual stimulation of any kind. Because the reach-to-grasp action was performed toward objects located in the same spatial position in the peripersonal space, the hand was transported always in the same direction, so the influence of the arm movement direction was identical during the execution of all grips. Moreover, the direction of gaze was kept constant during movement execution to avoid influencing the neuronal response. Finally, the orientation of the set of objects used for testing each neuron evoked grasps with similar wrist orientations, so that wrist orientation could not be a factor modulating the neuronal response. In conclusion, in our task, the only factor that could contribute to the differential discharge of the neurons was the grip type performed to acquire the object.

It could be argued that the reach component could be different for the different objects, despite the fact that the objects are placed in the same spatial position. If so, the observed neural modulation could be ascribed to the change in proximal aspects of reaching action rather than on changes in grip formation. According to our video-based estimation of proximal joint trajectories, the change in the position of the lesser tuberosity of the humerus was almost null among objects, and the changes in the position of the lateral epicondyle were confined within $1 \mathrm{~cm}$ while grasping different objects. This range of variation is too small to explain the strong neural modulations observed in this task.

\section{Grip selectivity in V6A population}

To examine the representation of grip types in the whole-cell population, we calculated the average normalized spike density functions for each one of the five grips tested (Fig. 3). The prominent feature of the population as a whole is that the temporal profile and intensity of discharge is similar for the five grip types (permutation test, $p>0.01$ ). In other words, there was no population bias for a specific grip. Individual neurons in V6A do show clear grip preferences (as shown in Fig. 2), but evidently these individual preferences compensate one another at the population level, so that all tested grips are approximately equally represented.

Many V6A neurons are modulated also by the appearance of the object at the beginning of each trial. Because this work focused on V6A discharges during the execution of reach-to-grasp of objects with different grips, a detailed analysis of the encoding of visual information related to objects is out of the scope of this paper. However, data on visual response are re- ported in supplemental data and Figure S1 (available at www. jneurosci.org as supplemental material).

To quantify the motor selectivity of V6A grasping neurons, we calculated a PI (see Materials and Methods) as well as the percentage discharge difference between the best and the second best grip and between the best and the worst grip. Figure 4 (left) shows that the PI of recorded cells ranged from 0.1 to 0.85 having an average of $0.39 \pm 0.17$, with the majority of neurons at intermediate PI values. The first two cells shown in Figure 2 have PI values of 0.34 and 0.37 , respectively, whereas the PI values of the remaining two was 0.71 and 0.81 , respectively.

Looking at the distribution histogram of the percentage discharge differences between the best and the second best grip (Fig. 4 , middle), it is evident that more than half of the neurons display a difference of $<20 \%$ (average, $20.6 \pm 17.9$ ). The percentage discharge difference between the best and the worst grip was of course $>20 \%$ (average, $56.7 \pm 18.3$ ) (Fig. 4, right), with some differences approaching $100 \%$ (see the cell shown in Fig. 2e). Figure 4 shows that the degree of selectivity to grip types changes from cell to cell and represents a continuum in V6A population.

Figure 5 shows that the population of V6A grasping neurons is able to discriminate between the different types of grip used to grasp the objects. The activity of each grasping neuron for each of the five tested grips was ranked in descending order to obtain the popula-

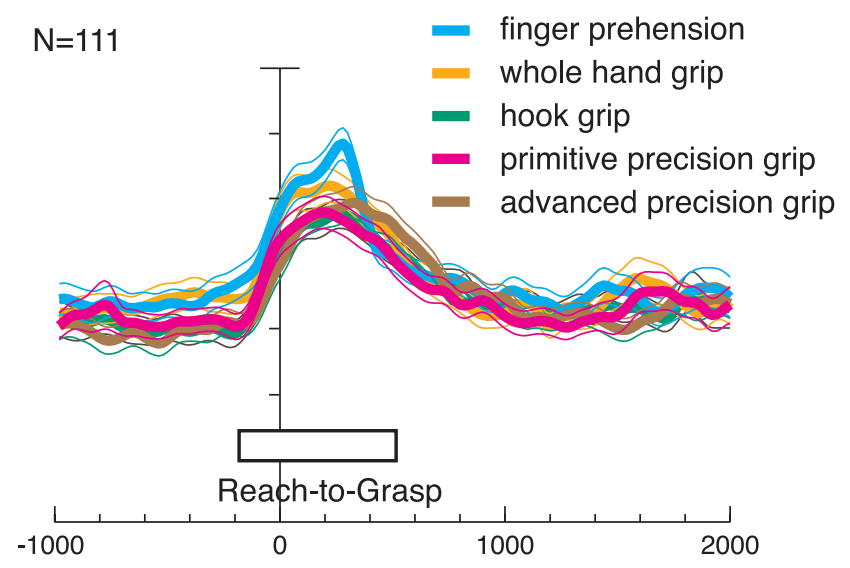

Figure 3. Absence of grip preference at population level. Activity of all task-related $111 \mathrm{~V} 6 \mathrm{~A}$ neurons for each tested grip (different colors) expressed as average normalized SDF (thick lines) with variability bands (SEM; light lines). Each cell of the population was taken into account five times, once for each grip type. Activity is aligned with the onset of movement. The five SDFs are not statistically different (permutation test), indicating an absence of a population preference for a specific grip in V6A. Scale on abscissa: $200 \mathrm{~ms} /$ division; vertical scale: $10 \%$ of normalized activity/division.
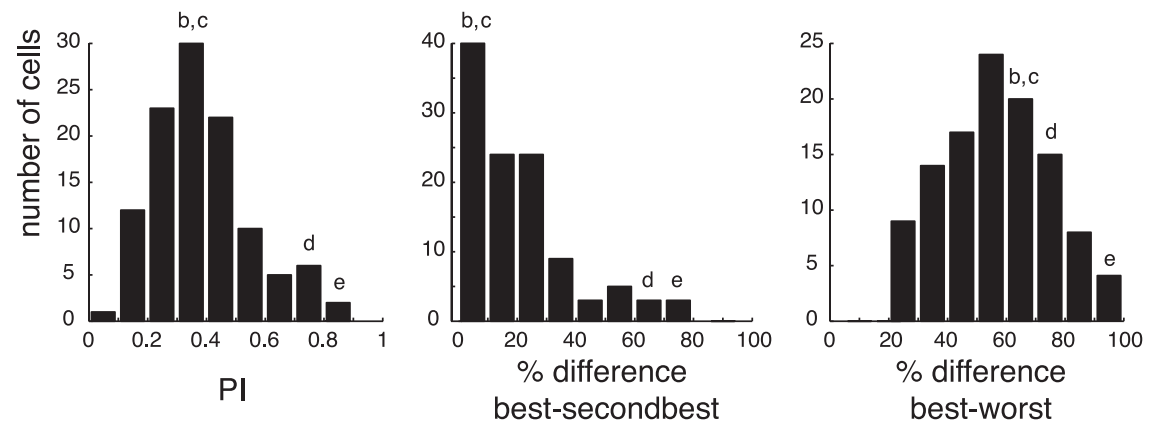

Figure 4. Motor selectivity of V6A neurons. Distribution of the preference index (left), percentage discharge differences between best and second best grip (middle), and percentage discharge differences between best and worst grip (right), during the reach-to-grasp epoch. Letters $\boldsymbol{b}$ - $\boldsymbol{e}$ above the histogram refer to the cells $\boldsymbol{b}-\boldsymbol{e}$ in Figure 2 , indicating which columns included those neurons. 
tion response for the best grip, the second best one, and so on, up to the fifth (worst) grip. This ranking was used to plot the neuronal discharge of the entire population independently of grip type. The plot shows a clear distinction among the activations during the execution of reach-to-grasp actions. The fact that the curves are already separated during the delay period, $\sim 1 \mathrm{~s}$ before the start of the movement, suggests that the neurons began to code the impending reach-to-grasp movement well before the occurrence of the movement itself. Just before the movement starts, the activity increases, and, as the movement evolves, the difference in activity among different grip types becomes more distinct, rising to the maximum before the end of the movement. The difference among the five curves is statistically significant during the reachto-grasp epoch (permutation test, $p<0.01$ ). This means that V6A population is able, before and during the execution of reach to grasp actions in darkness, to discriminate between the different grip types required to grasp the different objects.

To unravel the factors that determine the selectivity of the motor discharge, we performed hierarchical cluster analysis. The results are shown in Figure 6 . The analysis showed that the motor specificity of V6A neurons depends on the grip performed to grasp an object. Cluster analysis suggests that the use of the index finger is a critical factor. In fact, the hook grip, the primitive precision grip, and the advanced precision grip, for which the use

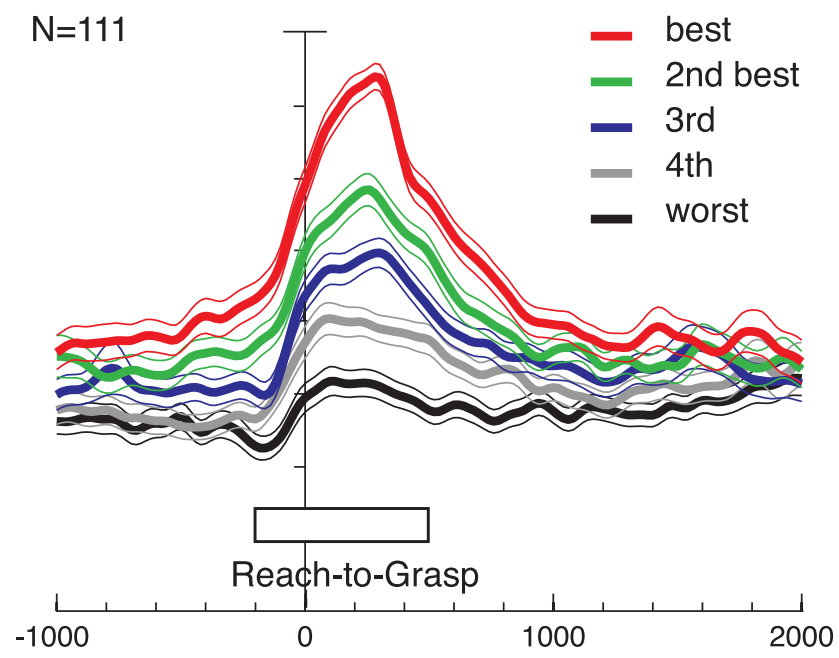

Figure 5. Grip discrimination in V6A. Population neuronal activity, expressed as averaged normalized SDFs, constructed by ranking the response of each neuron for each grip type in descending order according to the intensity of the response elicited in the reach-to-grasp epoch. Neuronal activities are aligned with the onset of movement. All responses are statistically different one from the other (permutation test, $p<0.01$ ), indicating that V6A grasp-related neurons are able to discriminate the different types of grips. All other details as in Figure 3. of the index finger is indispensable, form a strong cluster. Conversely, whole-hand and finger prehension, whose accomplishment does not specifically require the index finger, detach early from the other grips and form their own cluster.

\section{Discussion}

This study demonstrates that area V6A, a region of the medial posterior parietal cortex so far considered involved only in reaching, is also involved in grasping. In fact, we show that neural discharges of a significant number of V6A neurons vary according to the type of grip performed to grasp objects of different shapes.

Given that the aim of the present study was to investigate the motor aspects of area V6A, which contains visual neurons and receives visual inputs (Galletti et al., 1999; Gamberini et al., 2009), the task was performed in darkness to avoid the possibility that neural modulations observed during reach-to-grasp task were attributable to visual stimulations. Because V6A neurons code the direction of gaze (Galletti et al., 1995; Nakamura et al., 1999), the direction of arm reaching (Fattori et al., 2005), and the interaction between gaze and reach (Marzocchi et al., 2008), we kept constant both the direction of gaze and the spatial location of the target object, so the observed neural modulations could not be ascribed to these factors. Also, because recent evidence demonstrates that many V6A neurons are sensitive to wrist orientation during grasping (Fattori et al., 2009), the objects in the present study were oriented in such a way as to evoke grasps with a constant wrist orientation. In short, the only factor that varied and that could differentially influence the neuronal discharge was the shape of the object. Because the five objects we used required different grip types, the observed neuronal modulation during reach-to-grasp movements in darkness demonstrates that V6A cells are sensitive to hand preshaping and grip formation.

\section{Motor selectivity of V6A grasping neurons}

V6A neurons display preference for a particular grip or for a set of grips. In terms of selectivity, V6A grasping neurons appear to be as selective as grasping neurons in ventral premotor area F5, an area specifically involved in controlling grip formation (Raos et al., 2006). For movements made in the dark, F5 grasping neurons have a preference index of $0.42 \pm 0.2$ displaying a best/worst and best/second best percentage discharge difference of $63.6 \pm 2$ and $23.2 \pm 2$, respectively (V. Raos, M. A. Umiltà, V. Gallese, L. Fogasi, unpublished observations). In the same dark conditions, V6A grasping neurons show very similar preference indices $(0.39 \pm 0.2)$ and percentage discharge differences $(56.7 \pm 18.3$ and $20.6 \pm 17.9$, respectively).

The population behavior summarized in Figure 3 shows that V6A neurons do not prefer a specific type of grip, because all grips are equally represented, from the most rudimentary (whole-hand prehension) to the most skilled (advanced precision grip). However, the grasping population as a whole is able to discriminate among the different hand grips needed to grasp objects of widely differing shapes (Fig. 5). Cluster analysis revealed that the motor specificity of several V6A grasping neurons depends on the use of the index finger to grasp an object. This is in accordance with a psychophysical study demonstrating that, as the hand approaches an object, the index finger is primarily responsible for opening and closing the hand (Wing and Fraser, 1983).

\section{Figure 6. Clustering of grip types. Horizontal axis in the dendrogram indicates the distance coefficients at each step of the hierarchical clustering solution. Actual distances have been rescaled to the $0-25$ range. One cluster is formed by the hook grip together with the primitive and advanced precision grips. A second cluster, detaching early from the other cluster, is formed by whole hand and finger grips. The control of the index finger is essential for the grips in the first cluster but not for those in the second. \\ Objects Grip type \\ plate \\ groove \\ ball \\ handle \\ hook grip advanced precision grip whole-hand prehension finger prehension

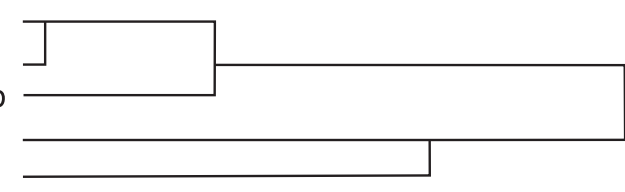


Results similar to those of V6A have been obtained from the cluster analysis of the movement-related discharge of grasping neurons in the anterior intraparietal area (AIP) and area F5, in which the determinant of the clustering was the pattern of hand grip (Murata et al., 2000; Raos et al., 2006). Unlike V6A, for F5 the most crucial factor for the distinction among grips was the use of the thumb (Raos et al., 2006).

\section{The dorsomedial parieto-frontal circuit is involved in grasping}

Area V6A belongs to the dorsomedial visual stream (Galletti et al., 2003), which is a subdivision of the dorsal visual stream, the neural pathway that processes visual information for action (Goodale and Milner, 1992; Milner and Goodale, 1995). Area V6A is connected with the middle intraparietal area (MIP) in the SPL (Shipp et al., 1998; Marconi et al., 2001; Gamberini et al., 2009), well known to be involved in coding reaching movements (Kalaska and Crammond, 1992; Johnson et al., 1996; Batista et al., 1999; Gail and Andersen, 2006; Pesaran et al., 2006), with areas of inferior parietal lobule as area AIP (Borra et al., 2008; Gamberini et al., 2009) involved in coding hand shape and orientation during grasping (Taira et al., 1990; Sakata et al., 1992; Gardner et al., 1999, 2007; Murata et al., 2000) and with sectors of dorsal premotor cortex (mainly area F2) (Matelli et al., 1998; Gamberini et al., 2009) devoted to code reaching but also grasping actions, as recently unraveled by Raos et al. (2004). Projections to V6A from area MIP [this latter being primarily coextensive with the socalled "parietal reach region" (Gail and Andersen, 2006; Pesaran et al., 2006)] could provide input for directing the arm, whereas those arising from AIP could allow the appropriate grip to be selected according to the object shape and size.

A recent study has demonstrated that area F2, in addition to a proximal forelimb field, contains a distal forelimb field whose electrical microstimulation evoked wrist and finger movements (Raos et al., 2003). In agreement with these data, single-neuron recordings in F2 have revealed the presence of cells selective for wrist orientation and for the type of grip required for grasping an object (Raos et al., 2004). It appears therefore that the dorsomedial parieto-frontal pathway V6A-F2 is able to control proximal as well as distal aspects of prehension movements. It could form a reverberating circuit controlling the entire act of prehension from planning through to execution, including self-correction adjustments.

Given the established involvement of the AIP-F5 dorsolateral parieto-frontal pathway in grasping control (Jeannerod et al., 1995), it is reasonable to wonder why a second route dealing with the same function (grasping) would exist in the primate brain. Actually, AIP neurons encode the type of grasping used to acquire objects (Taira et al., 1990; Sakata et al., 1992; Murata et al., 2000), but the activity of these neurons is not influenced by the change in position of the object in space (Taira et al., 1990; Gardner et al., 2007). In agreement with these functional data, lesion studies in both monkeys and humans have suggested that AIP is involved in the control of grasping but not of reaching (Gallese et al., 1994; Binkofski et al., 1998) (for a recent review on this subject, see Castiello and Begliomini, 2008). Conversely, a plethora of studies shows that the discharge of neurons in SPL areas is modulated by the direction of reaching. It is now known that neurons in one of these areas (V6A) are also modulated by the orientation of the wrist (Fattori et al., 2009) and, as the present data demonstrate, by grip formation. Therefore, although AIP is crucial to control distal movements such as those required for grasping and manipulating objects, V6A is likely involved in the control of both reaching and grasping phases, that is the entire act of prehension. This hy- pothesis is supported by lesion studies in monkeys: selective, bilateral ablation of area V6A evoked misreaching but also misgrasping and incorrect wrist orientation (Battaglini et al., 2002).

Whereas the dorsolateral AIP-F5 stream may adapt motor behavior to current perceptual conditions by integrating information processed in the ventral stream into the prehension plan (Rizzolatti and Matelli, 2003), the dorsomedial fronto-parietal network V6A-F2 could be used particularly when fast control is required (Galletti et al., 2003; Fattori et al., 2009). This hypothesis finds support in recent fMRI experiments in humans, which suggest that the dorsolateral stream may incorporate perceptual information into its sensorimotor transformations, whereas the dorsomedial circuit may support motor planning for prehension on the basis of advance visual information (Grol et al., 2007; Verhagen et al., 2008). These data, together with the present ones, suggest new ways forward for developing computational models of visuomotor control in the context of the wider question of how the brain organizes our visually guided actions in general.

\section{Conclusions}

We demonstrate here that the dorsomedial visual pathway, and in particular the medial posterior parietal area V6A, has a role not only in controlling the transport of the hand to the location of the desired object but also in shaping the hand to match the physical features of the object. Our data thus indicate that area V6A is part of a unified sensorimotor network involved in the control of the hand while approaching and interacting with objects during reach-to-grasp actions.

\section{References}

Arbib MA (1981) Perceptual structures and distributed motor control. In: Motor control, Handbook of physiology, Sec 1, The nervous system (Brooks VB, ed), pp 1449-1480. Baltimore: Williams and Wilkins.

Batista AP, Buneo CA, Snyder LH, Andersen RA (1999) Reach plans in eyecentered coordinates. Science 285:257-260.

Battaglini PP, Muzur A, Galletti C, Skrap M, Brovelli A, Fattori P (2002) Effects of lesions to area V6A in monkeys. Exp Brain Res 144:419-422.

Binkofski F, Dohle C, Posse S, Stephan KM, Hefter H, Seitz RJ, Freund HJ (1998) Human anterior intraparietal area subserves prehension: a combined lesion and functional MRI activation study. Neurology 50:1253-1259.

Borra E, Belmalih A, Calzavara R, Gerbella M, Murata A, Rozzi S, Luppino G (2008) Cortical connections of the macaque anterior intraparietal (AIP) area. Cereb Cortex 18:1094-1111.

Caminiti R, Ferraina S, Johnson PB (1996) The sources of visual information to the primate frontal lobe: a novel role for the superior parietal lobule. Cereb Cortex 6:319-328.

Castiello U, Begliomini C (2008) The cortical control of visually guided grasping. Neuroscientist 14:157-170.

Desmurget M, Prablanc C, Arzi M, Rossetti Y, Paulignan Y, Urquizar C (1996) Integrated control of hand transport and orientation during prehension movements. Exp Brain Res 110:265-278.

Fattori P, Gamberini M, Kutz DF, GallettiC (2001) 'Arm-reaching' neurons in the parietal area V6A of the macaque monkey. Eur J Neurosci 13:2309-2313.

Fattori P, Kutz DF, Breveglieri R, Marzocchi N, Galletti C (2005) Spatial tuning of reaching activity in the medial parieto-occipital cortex (area V6A) of macaque monkey. Eur J Neurosci 22:956-972.

Fattori P, Breveglieri R, Marzocchi N, Filippini D, Bosco A, Galletti C (2009) Hand orientation during reach-to-grasp movements modulates neuronal activity in the medial posterior parietal area V6A. J Neurosci 29:19281936.

Gail A, Andersen RA (2006) Neural dynamics in monkey parietal reach region reflect context-specific sensorimotor transformations. J Neurosci 26:9376-9384.

Gallese V, Murata A, Kaseda M, Niki N, Sakata H (1994) Deficits of hand preshaping after muscimol injection in monkey parietal cortex. Neuroreport 5:1525-1529.

Galletti C, Battaglini PP, Fattori P (1995) Eye position influence on the 
parieto-occipital area PO (V6) of the macaque monkey. Eur J Neurosci 7:2486-2501.

Galletti C, Fattori P, Kutz DF, Gamberini M (1999) Brain location and visual topography of cortical area V6A in the macaque monkey. Eur J Neurosci 11:575-582.

Galletti C, Kutz DF, Gamberini M, Breveglieri R, Fattori P (2003) Role of the medial parieto-occipital cortex in the control of reaching and grasping movements. Exp Brain Res 153:158-170.

Gamberini M, Passarelli L, Fattori P, Zucchelli M, Bakola S, Luppino G, Galletti C (2009) Cortical connections of the visuomotor parietooccipital area V6Ad of the macaque monkey. J Comp Neurol 513:622-642.

Gardner EP, Ro JY, Debowy D, Ghosh S (1999) Facilitation of neuronal activity in somatosensory and posterior parietal cortex during prehension. Exp Brain Res 127:329-354.

Gardner EP, Babu KS, Reitzen SD, Ghosh S, Brown AS, Chen J, Hall AL, Herzlinger MD, Kohlenstein JB, Ro JY (2007) Neurophysiology of prehension. I. Posterior parietal cortex and object-oriented hand behaviors. J Neurophysiol 97:387-406.

Goodale MA, Milner AD (1992) Separate visual pathways for perception and action. Trends Neurosci 15:20-25.

Grol MJ, Majdandzić J, Stephan KE, Verhagen L, Dijkerman HC, Bekkering H, Verstraten FA, Toni I (2007) Parieto-frontal connectivity during visually guided grasping. J Neurosci 27:11877-11887.

Jeannerod M (1997) The cognitive neuroscience of action. Oxford: Blackwell.

Jeannerod M (1981) Intersegmental coordination during reaching at natural visual objects. In: Attention and performance (Long J, Baddeley A, eds), pp 153-168. Hillsdale, NJ: Erlbaum.

Jeannerod M, Arbib MA, Rizzolatti G, Sakata H (1995) Grasping objects: the cortical mechanisms of visuomotor transformation. Trends Neurosci 18:314-320.

Johnson PB, Ferraina S, Bianchi L, Caminiti R (1996) Cortical networks for visual reaching: physiological and anatomical organization of frontal and parietal lobe arm regions. Cereb Cortex 6:102-119.

Kalaska JF, Crammond DJ (1992) Cerebral cortical mechanisms of reaching movements. Science 255:1517-1523.

Krakauer J, Ghez C (2000) Voluntary movement. In: Principles of neural science, Ed IV (Kandel ER, Schwartz JH, Jessel TM, eds). New York: McGraw-Hill.

Kutz DF, Marzocchi N, Fattori P, Cavalcanti S, Galletti C (2005) Real-time supervisor system based on trinary logic to control experiments with behaving animals and humans. J Neurophysiol 93:3674-3686.

Luppino G, Murata A, Govoni P, Matelli M (1999) Largely segregated parietofrontal connections linking rostral intraparietal cortex (areas AIP and VIP) and the ventral premotor cortex (areas F5 and F4). Exp Brain Res 128:181-187.

Luppino G, Hamed SB, Gamberini M, Matelli M, Galletti C (2005) Occipital (V6) and parietal (V6A) areas in the anterior wall of the parieto-occipital sulcus of the macaque: a cytoarchitectonic study. Eur J Neurosci 21: 3056-3076.

Marconi B, Genovesio A, Battaglia-Mayer A, Ferraina S, Squatrito S, Molinari M, Lacquaniti F, Caminiti R (2001) Eye-hand coordination during reaching. I. Anatomical relationships between parietal and frontal cortex. Cereb Cortex 11:513-527.
Marzocchi N, Breveglieri R, Galletti C, Fattori P (2008) Reaching activity in parietal area V6A of macaque: eye influence on arm activity or retinocentric coding of reaching movements? Eur J Neurosci 27:775-789.

Matelli M, Govoni P, Galletti C, Kutz DF, Luppino G (1998) Superior area 6 afferents from the superior parietal lobule in the macaque monkey. J Comp Neurol 402:327-352.

Milner AD, Goodale MA (1995) The visual brain in action. Oxford: Oxford UP.

Mon-Williams M, McIntosh RD (2000) A test between two hypotheses and a possible third way for the control of prehension. Exp Brain Res 134:268-273.

Moody SL, Zipser D (1998) A model of reaching dynamics in primary motor cortex. J Cogn Neurosci 10:35-45.

Murata A, Gallese V, Luppino G, Kaseda M, Sakata H (2000) Selectivity for the shape, size, and orientation of objects for grasping in neurons of monkey parietal area AIP. J Neurophysiol 83:2580-2601.

Nakamura K, Chung HH, Graziano MS, Gross CG (1999) Dynamic representation of eye position in the parieto-occipital sulcus. J Neurophysiol 81:2374-2385.

Pesaran B, Nelson MJ, Andersen RA (2006) Dorsal premotor neurons encode the relative position of the hand, eye, and goal during reach planning. Neuron 51:125-134.

Raos V, Franchi G, Gallese V, Fogassi L (2003) Somatotopic organization of the lateral part of area F2 (dorsal premotor cortex) of the macaque monkey. J Neurophysiol 89:1503-1518.

Raos V, Umiltá MA, Gallese V, Fogassi L (2004) Functional properties of grasping-related neurons in the dorsal premotor area F2 of the macaque monkey. J Neurophysiol 92:1990-2002.

Raos V, Umiltá MA, Murata A, Fogassi L, Gallese V (2006) Functional properties of grasping-related neurons in the ventral premotor area F5 of the macaque monkey. J Neurophysiol 95:709-729.

Rizzolatti G, Matelli M (2003) Two different streams form the dorsal visual system: anatomy and functions. Exp Brain Res 153:146-157.

Sakata H, Taira M, Mine S, Murata A (1992) The hand-movement-related neurons of the posterior parietal cortex of the monkey: their role in the visual guidance of hand movement. In: Control of arm movement in space: neurophysical and computational approaches (Experimental Brain Research Series 22), pp 185-198. Berlin: Springer.

Shipp S, Blanton M, Zeki S (1998) A visuo-somatomotor pathway through superior parietal cortex in the macaque monkey: cortical connections of areas V6 and V6A. Eur J Neurosci 10:3171-3193.

Smeets JB, Brenner E (1999) A new view on grasping. Motor Control 3:237-271.

Taira M, Mine S, Georgopoulos AP, Murata A, Sakata H (1990) Parietal cortex neurons of the monkey related to the visual guidance of hand movement. Exp Brain Res 83:29-36.

Verhagen L, Dijkerman HC, Grol MJ, Toni I (2008) Perceptuo-motor interactions during prehension movements. J Neurosci 28:4726-4735.

Wing AM, Fraser C (1983) The contribution of the thumb to reaching movements. Q J Exp Psychol A 35:297-309.

Wise SP, Boussaoud D, Johnson PB, Caminiti R (1997) Premotor and parietal cortex: corticocortical connectivity and combinatorial computations. Annu Rev Neurosci 20:25-42. 